\title{
تطوير مواصفات الطابوق الناري العازل المستعمل في تبطين افران الصناعة النفية
} Development the Specifications of the light Weight fire brick

\author{
عبدالكريم دهش الزبيدي ،جامعة النهرين - كلية الهندسة
}

الخلاصة:

$$
\begin{aligned}
& \text { تم في هذا البحث انتاج طابوق ناري عازل خفيف ذو } \\
& \text { مو اصفات عالية من اضافة مادة فلوريد الالمنيوم }
\end{aligned}
$$

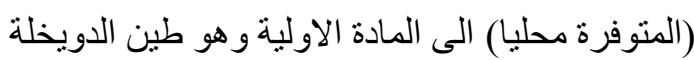

$$
\begin{aligned}
& \text { تم الحرق في درجات حرارة عالية ومختلفة وتتراوح } \\
& \text { من } 900 \text { - إلى 1300مْ حيث تحدث تفاعلات } \\
& \text { كيماوية بين الطين و المادة }
\end{aligned}
$$

الكيماوية المضافة حيث يتكون نتيجة التفاعل لهين

المو لايت المسامي و التي تزيد من قابلية هذا النوع من لـانه

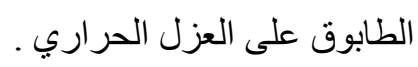

ميزات الطابوق المنتج في هذا البحث هذا هو

$$
\text { انخفـاض معامـل التوصيل الحراري }
$$

(0.15kcal/m.h.c) المرجع2 و المرجع 3هي عبارة عن بحوث قام بها مجمو عة من الباحثين في مركز بحوث البناء التابع

لوزارة الاسكان حاليا وسابقا الى مجلس البحث لبث العلمي.

\section{The Abstract :}

In this research alight weight

insulating brick was produced with high specifications by adding aluminum fluoride \{which is available in Iraq $\}$ to Dwechla clay. after that the mixture is fired in high differentTemperatures reaching 1300 C. Chemical reaction happens during burning which results $\mathrm{sp}$ onge mullite increasing the thermal insulation of the refractory or the brick. In this research law heat conduction of the insulating brick $\{0.15 \mathrm{kcal} / \mathrm{m} . \mathrm{h} . \mathrm{c}\}$ was produced.

المقدمة:

للعو ازل الحرارية السير اميكية استعمالات كثيرة منها على سبيل المثال لا الحصر استعمالها لتبطين الافران في العديد من الصناعات المختلفة والتي تتطلب درجات حرارية عالية جدا تصل الى اكثر من 1000

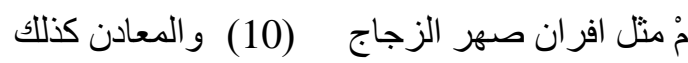
الافر ان المستعملة في الصناعات البتروكيمياوية وكذلك في تبطين المر اجل البخارية و وابر اج تصفية النفط وفائدة تبطين الافران بهذا النوع من العوازل

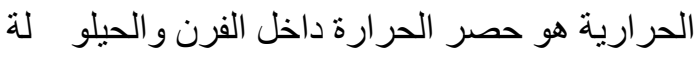

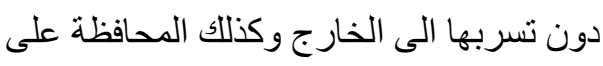
الغلاف الخارجي كما ان تبطين الافران بهذا النوع من العو ازل الحر ارية يحافظ على حرارة معتدلة وملائمة للعمل في المحيط الخارجي افرن ـ ويستعمل حوالي 79\% من صناعة الحديد و الصلب هذا النوع من الطابوق[2]. ان الخو اص الفيزياوية و الميكانيكية للطابوق الناري الطيني تعتمد على مكونات الطين الكيمياوية و المعدنية , الجدولان رقم (1) و و(2) يوضحان التحاليل, الكيماوية لهذا الطين الذي تم الحصول عليه من (منطقة الدويخلة محافظة الانبار ) تزداد قابلية

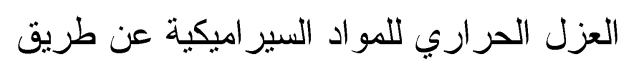


تخفيض كثافة المادة وان الطريقة الاكثر استعمالا داخل المادة ـ و هذه الفر اغات او المسامات يجب ان تكون صغيرة الحجم بما فيه الكفاية وذللك لمنع حدوث تيار ات حمل حرارية [1] وذللك لتقليل قابلية التوصيل الحر اري وجعلها و اطئة ق ياسا مع المو اد

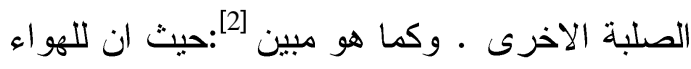
قابلية توصيل حراري هي 0.029kcal/m.h.C للطين المحترق الصلب 0.45kcal/m.hC

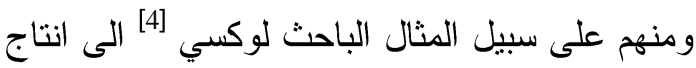
مو اد عازلة حر ارية سير اميكية باضافة مو اد غير عضوية الى الطين وقدمت هذه البحوث نتائج مشجعة وتتيح للاخرين الفرصة في تطوير هذه البحوث. مع امكانية تطبيقها على الاطيان العر اقية. علما ان التركيب المعدني والكيماوي للاطيان العر اقية يختلف عما هو في اطيان البلدان الاخرى و التي اجريت فيها البحوث السابقة .

\section{طرق انتاج الطابوق الناري العازل الخفف :} توجد عدة طرق لانتاج هذه الطابوق ولكن اهمها تلك [4]

التي تخص هذا البحث هو ما عرضه جي البرت و الذي يتضمن انتاج طابوق ناري عازل خفيف طيني وذللك باضافة مركبات الالمنيوم مثل اوكسيد و هيدروكسيد وفلوريد الالمنيوم .ان البحث الحالي يعتمد على تطبيق هذه المفاهيم .ان تفاعل فلوريد

الالمنيوم مع السليكا قد صور حسب ما عرضده

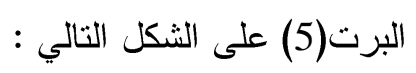

$12 \mathrm{AlF}_{3}+13 \mathrm{SiO}_{2}-----\rightarrow 2\left\{3 \mathrm{Al}_{2} \mathrm{O}_{3} \cdot 2 \mathrm{SiO}_{2}\right\}+9 \mathrm{SiF}_{4}----$ (1)
في هذا المجال هو تكوين فر اغات هو ائية او غازية حيث ان الناتج النهائي لمثل هذا التفاعل هو كاوؤلين(Kaoline) مع امكانية تحرر غاز Sتاركا ور اءه فقاعات غازية تساعد على زيادة المسامية للطابوق المنتج و هناك تصور ثاني للتفاعل. $3 \mathrm{SiO}_{2}+4 \mathrm{AlF}_{3}-----\mathrm{-}_{2} \mathrm{Al}_{2} \mathrm{O}_{3}+3 \mathrm{SiF}_{4}$

كما في التفاعل رقم (1) يكون ناتج تفاعل رقم (2) غاز فلوريد السليكون SiF4 ايضا معطيا النتيجة نفسها من حيث تر كه فقاعات غازية تساعد على زيادة مسامية الطابوق مع نتيجة نهائية زيادة العزل الحراري للطابوق المنتج ان مادة فلوريد الالمنيوم مادة متسامية, ان اضافة فلوريد الالمنيوم الى الطين (طين الدويخلة) يكوّن على الاغلب غاز فلوريد الهيدروجين او فلوريد السيلكون SiF4 (HF)

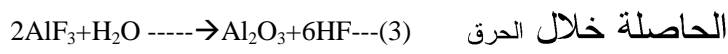
$\mathrm{SiO}_{2}+4 \mathrm{HF}$------ $\rightarrow \mathrm{SiF}_{4}+2 \mathrm{H}_{2} \mathrm{O}-----(4)$

\section{المادة الاولية(Clay)}

المادة الاولية المستخدمة في هذا البحث هي اطيان

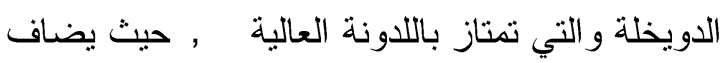
اليها مادة فلوريد الالمنيوم وكذلك اوكسيد وهيدروكسيد

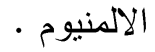

ملاحظة : ان مادة فلوريد الالمنيوم منوفرة وتصنع.

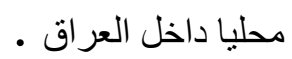
طريقة العمل والاجهزة المستعملة: 1 1 العمل: 1 تم اجر اء التجارب على نماذج من اطيان الدويخلة ومو اد كيماوية مثل فلوريد واوكسيد وهيدروكسيد الالمنيوم 


\section{2-تحضير المواد الاولية}

تم اجر اء عملية التكسير على الطين المستعمل ثم الطحن و الغربلة للحصول على طين ناعم باستعمال غربال من اهن نوع (Mesh 60) ام| المواد الكيمياوية فقد اجريت عليها عمليات طحن و غربلة ثم التجفيف للحصول على عجينة متجانسة عند عمل الطابوق , اما الطين المحروق فقد تم تحضيره بحرق كمية من الطين في فرن كهربائي تصل حر ارته الى 1450 مُولمدة ساعنين .

\section{3-تحضير النماذج:}

1 - تحضير العجينة: نم تحضير العجينة بخلط نسب

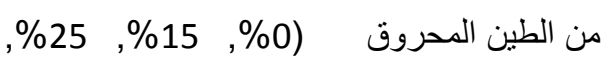
35\%) مع الطين الغير محروق بنسب (50\%) \% \% ثم بعد ذلك تضاف احدى المواد الكيمياوية مثل هيدروكسيد الالمنيوم الى الخليط الطيني

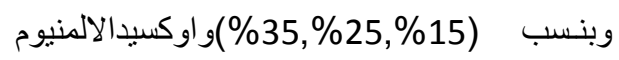

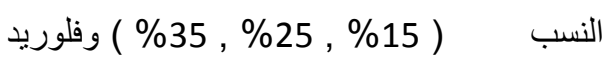

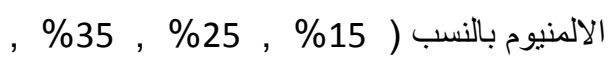

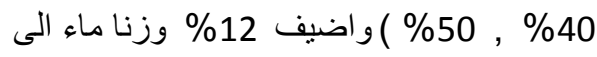
العجينة محسوبا من وزنها الكلي حيث اختبرت هذه النسبة من الماء تجريبيا حيث وجد ان الطين

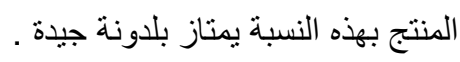
2 - التشكيل : لغرض تحضير النماذج المختبرية تم اختيار طريقة الكبس ذو الرطوبة الجزئية وذللك لان الطابوق المنتج بهذه الطريقة يمتاز بمسامية جيدة احسن من الطرق الاخرى وتقلص جاف منخفض وقوة تحمل عالية بالاضافة الى مقاو مته للتغير ات الحرارية الفجائية ان العينة الناتجة من جهاز التشكيل اليدوي تكون على شكل اسطو اني قطره 32.5 ملم ـ لقد تم تبني طريقة الكبس ذو الرطوبة
الجزئية وذللك لسهولتهاولتوفر الاجزة المختبرية (المكبس اليدوي مثل عصلارة الفو اكه المستعملة في محلات بيع العصائر). 3. التجفيف : الماء المخلوط مع الطين الحراري

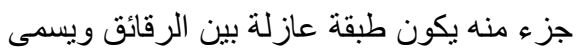

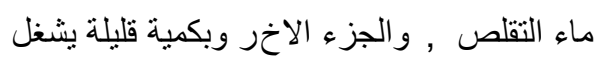

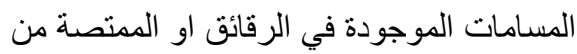
قبل سطح الرقائق ويسمى بماء المسامات و اثثاء عملية التجفيف تثقلص النماذج نتيجة لخروج الماء

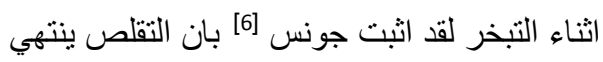
عندما يخرج كل ماء التقلص وكذلك روبنكيكوف[7] اكد ما ذكره ج ونس ويعطي

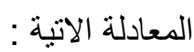

Total Water $=$ Shrinkage Water + Pores Water

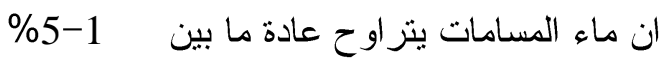
من وزن النموذج الجاف ويتبخر اثثاء عملية التسخين وبدرجة 110 مجز السير اميكية يتتاسب طرديا مع كمية الماء المفقودة كلما كانت اللدو نة عالية كلما كانت الرقائق صغيرة الحجم كلما ازدادت كمية ماء التقلص وبالتالي يؤدي الى زيادة تقلص المادة السير اميكية

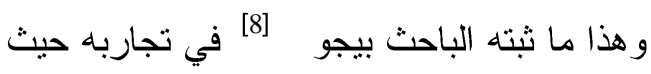
توصل الى علاقة بين التقلص و اللدونة ووجد بأنه

كلما ازدادت اللاونة ازداد التقلص و اعطى لهذه العلاقة الهعادلة التالية : - التالية

Plasticity $=($ shrinkage water/total water $) *$ linear shrinkage

من هذا المنطلق ولتفادي حساسية الطابوق المكبوس اثثاء التجفيف تم اختيار حجم الرقائق ومن ثم التجفيف في درجة حرارة 110ْم ومدة التجفيف هو احد عو امل

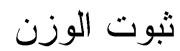


المرحلة الاولى:

وضع العينات في الجو الطلق ولمدة 48 ساعة لكي يتم

التبخر طبيعيا .

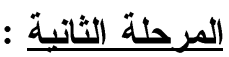

تجفيف العينات بالتسخين الكهربائي وفي درجة حرارة

60 مْ ولمدة 24 ساعة ثم رفع الحرارة لتصل الى بلى

$$
110 \text { مْ ولمدة } 24 \text { ساعة اخرى . }
$$

1 -الحرق : عند تسخين الاطبان من الكائولينات

يبدأ بفقدان الماء الميكانيكي و المتبقي (kaolinite)

بعد التجفيف وكذللك ماء التبلور الباحث

راشيفسكي][9] لاحظ بان اكبر كمية من الماء

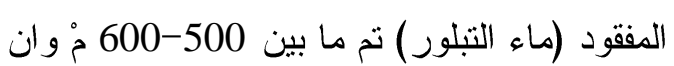

فقدان الماء يؤدي الى ظهور التقلص و الذي يبدأ في درجة حرارة 500 مْ ويتتهي في درجة حرارة 800 مْ وكما موضح في الثكل رقم هذا البحث تم الحرق في فرن كهربائي وفي درجات حر ارية مختلفة تتراوح من . 1300

الفحوصات

تم اجر اء الفحوصات المختبرية على الطابوق المنتج في هذا البحث لغرض مقارنة خو اصه مع المو اصفات

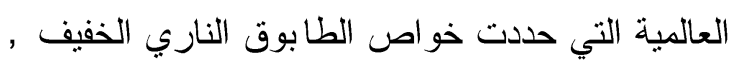
حيث تتراوح الكثافة من 600 الى 1600 كغم I م3 3 مسامية 25 الى 70 \% و التحمل من 20 الى 25 كغم

$$
1 \text { اسم2[2] } 1 \text { فحص الكثافة الكلية }
$$

توزن العينة وهي جافة ومن ثم تغطس في الماء

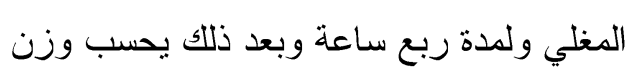

العينة المشبعة بالماء وكالاتي :
الكثافة الكلية = وزن النموذج الجاف بعد الحرق ا وزن النموذج المشبع بالماء - وزن النموذج بعديه بالماء غم 1 سم3 ـ الرسم (2) يمثل نتائج هذا البحث .

2

تم تحديد نسبة الماء الممنص من قبل النموذج كالاتي : نسبة امتصاص الماء = وزن النموذج المشبع بالماء - وزن النموذج الجاف ل وزن النموذج الجاف 100*

\section{3 فحص قوة التحمل :}

لغرض قياس قوة تحمل الطابوق الخفيف تم ايجاد المساحة السطحية للعينات وثم تسليط ضغط على لمري العينات بواسطة جهاز ضغط هيدروليكي يدوي حتى الانكسار الذي يحدث في العينة : قوة التحمل = الحمل المساحة السطعبة كغم اسم2 2

\section{4 نحص التوصيل الحراري:} تم ايجاد التوصيل الحراري لجميع النماذج وفي درجات حرارية مختلفة باستعمال الرسم رقم (4)

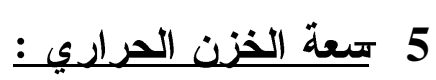

تم قياس سعة الخزن الحراري للطابوق الخفيف في هذا البحث باستعمال العلاقة التالية[1]:

Heat capacity $=\sum_{i=1}^{n} Y_{i} X_{i} C_{i}$

$$
\begin{aligned}
& \text { Yi = النسبة الوزنية } \\
& \text { الكثافة }
\end{aligned}
$$

[2] C C السعة الحر ارية 


\section{6}

المسامية = وزن النموذج المشبع بالماء -وزن

النموذج الجاف ل اوزن النموذج المشبع بالماء وزن النموذج بالماء * 100 مع الاخذ بنظر الاعتبار ان بعض مادة فلوريد الالمنيوم يذوب

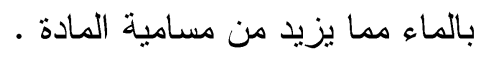
7

تم قياس لدونة الطين المستعمل حسب طريقة وتتم العملية بأعداد خمس نماذج (pfeffer) اسطو انية الثكل مع خلط الطين الجاف مع كميات مخلفة من الماء بو اسطة قالب له طول قياسي مقداره 40 ملم مرفق مع الجهاز وتوضع النماذج في قاعدة الجهاز ويسلط عليها نقل مقداره 1192 غم ويقاس طول النموذج بعد تسليط الضغط وفي نفس الوقت تؤخذ كمية من كل عينة توضع في جفنة ويقاس وزنها الرطب وتجفف بعد ذلك حتى لهى لهن ثبوت وزن العينة ومن ثم قياس وزنها الجاف : $\mathrm{a}=$ ho / h1 where ho= height of the sample before loading $\mathrm{mm}$ And h1 = height of the sample after loading

$\mathrm{W} \%=(\mathrm{G} 1-\mathrm{G} 2 / \mathrm{G} 1) * 100$

Where $\mathrm{W}=$ water content

G1 = weight of the moist sample in gm

$$
\begin{aligned}
& \mathrm{G} 2 \text { = weight of the dried sample } \\
& \text { in } \mathrm{gm}
\end{aligned}
$$

$$
\begin{aligned}
& \text { الثكل البياني رقم (6) يمثل نتائج هذه التجربة } \\
& \text { بالتسبة للطين المحروق وغير المحروق. }
\end{aligned}
$$

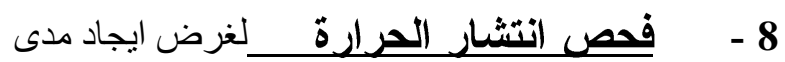

$$
\begin{aligned}
& \text { انتشار الحرارة في الطابوق الخفيف المنتج في هذا } \\
& \text { البحث فقد استعملت العلاقة التالية[1]. }
\end{aligned}
$$

Thermal Diffusivity $=$ Thermal conductivity / heat storage capacity in $\mathrm{m} 2 / \mathrm{h}$

While thermal conductivity in kcal / h.m.c

\section{الاستتتاجات :}

$$
\begin{aligned}
& 1 \text { - الكثافة : تم الحصول على طابوق ذو كثافة واطئة تصل } \\
& \text { الى 67و. غم ل سم3 نتيجة اضافة مادة فلوريد }
\end{aligned}
$$

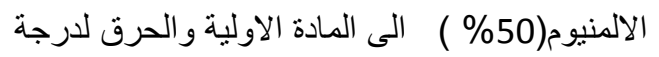

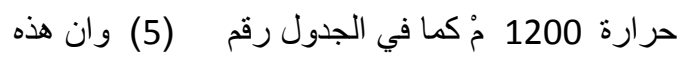

$$
\begin{aligned}
& \text { النتيجة التي تم الحصول عليها في هذا البحث هي ضمن } \\
& \text { المو اصفات العالمية للطابوق الناري الخفيف حيث }
\end{aligned}
$$

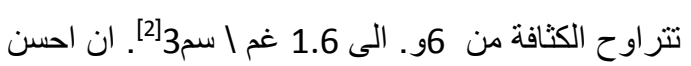

$$
\begin{aligned}
& \text { نتيجة نم الحصول عليها هي اضافة مادة فلوريد الالمنيوم } \\
& \text { الى الطين وحرقها بدرجة حرارة } 1200 \text { مُ . } \\
& 2 \text { - المسامية : في هذا البحث تم الحصول على مسامية } \\
& \text { للطابوق تتراوح من 13,8 الى16\%,5 ومن الثكل رقم } \\
& \text { (3) يتضح ان المسامية تزداد مع ازدياد الحرارة للحرق } \\
& \text { ولكن هذه العلاقة تبقى الى درجة حرارة } 1200 \text { خ حيث } \\
& \text { بعد ذلك تتخفض المسامية كلما ارتفعت د رجة الحرارة } \\
& \text { اكثر من 1200مْ كما في الجدول رقم (3) و السبب في } \\
& \text { ذلك يعود الى انصهار حبيبات الطين في الحرارات } \\
& \text { العالية جدا وبالتالي امتلاء المسامات بتلك المنصهرات }
\end{aligned}
$$


3 - قيوة التحمل : ان الثكل رقم (4) بيين العلاقة بين حرارة

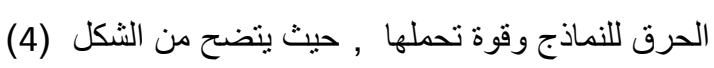

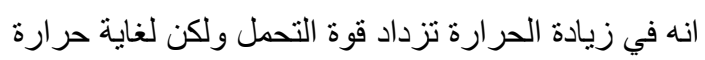
1000 مُحيث بعد ذلك تبدأ بالنزول كما في الجدول رقم

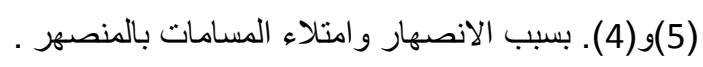
4

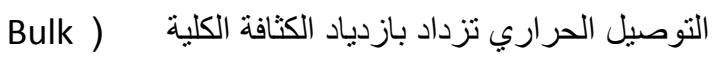
للطابوق الناري الخفيف حيث كلما استطعنا (density خفض كثافة الطابوق المنتج كلما قلت قابلية التوصيل الحراري أب زيادة العزل الحراري للطابوق الناري الخفيف المنتج في هذا البحث. ومن الجدول رقم (9) يتضح انه ادنى قيمة يمكن الحصول عليها هي (15, 0) وفي حرارة 1200مْ ومن اضافة مادة فلوريد الالمنيوم

$$
\text { وبالمقارنة مع المو اد الاخرى كما في الجداول }
$$

5 = سعة الخزن الحرارى : :ومن الجدول (10) نلاحظ بان

الطابوق الناري الطيني المنتج في هذا البحث له قابلية عزل

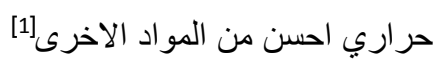

6 كلادونة : من الثكل رقم (6) يتضح ان احسن نسبة للماء

a = $3.3 \quad$ المضاف يمكن الحصول عليها عندما تكون

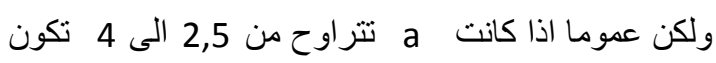
العجينة سهلة النشكيل , في الثكل (6) الخط الدستقيم رقم (1) يمثل الطين غير المحروق الذي يمتاز باللدونة العالية ولكن هذه اللدونة تتخفض باضافة الطين المحروق كما هو

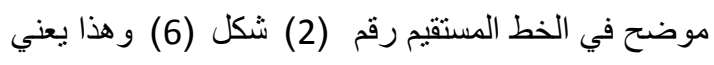

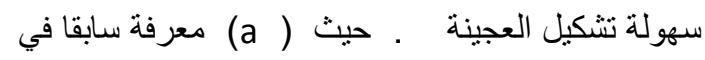
الصفحة رقم(5) من هذا البحث .

\section{Table (1) : - Chemical analysis of Dewechla clay}

\begin{tabular}{|c|c|c|}
\hline Material & $\%$ & $\begin{array}{c}\text { Indicated limits for suita- } \\
\text { ble fire clays }\end{array}$ \\
\hline $\mathrm{Al} 2 \mathrm{O} 3$ & 37 & \\
\hline $\mathrm{SiO} 2$ & 57.8 & Up to $4 \%$ \\
\hline $\mathrm{TiO} 2$ & 1.6 & Not more than $2.5 \%$ \\
\hline $\mathrm{Fe} 2 \mathrm{O} 3$ & 1.45 & Not more than $1 \%$ \\
\hline $\mathrm{CaO}$ & 0.7 & Not more than $3 \%$ \\
\hline $\mathrm{K} 2 \mathrm{O}$ & 0.4 & \\
\hline
\end{tabular}


Table (2) :Mixture of clay and Al2O3

\begin{tabular}{|c|c|c|c|c|c|c|c|}
\hline $\begin{array}{c}\text { Firing } \\
\text { Temp } \\
\text { C. }\end{array}$ & $\begin{array}{c}\text { Content } \\
\text { of clay } \\
\%\end{array}$ & $\begin{array}{c}\text { Content } \\
\text { of burn- } \\
\text { ing clay } \\
\%\end{array}$ & $\begin{array}{c}\text { Content } \\
\text { of } \\
\text { Al2O3 } \\
\%\end{array}$ & $\begin{array}{c}\text { Bulk } \\
\text { density }\end{array}$ & $\begin{array}{c}\text { Porosity } \\
\%\end{array}$ & $\begin{array}{c}\text { Water ab- } \\
\text { sorption } \\
\%\end{array}$ & $\begin{array}{c}\text { Compressive } \\
\text { strength } \\
\mathrm{kg} / \mathrm{cm} 2\end{array}$ \\
\hline 900 & 50 & 15 & 35 & 1.3 & 49.95 & 38.4 & - \\
\hline & 50 & 25 & 25 & 1.34 & 46.88 & 34.94 & - \\
\hline & 50 & 35 & 15 & 1.37 & 44 & 32 & - \\
\hline 1000 & 50 & 15 & 35 & 1.24 & 50.1 & 40.55 & - \\
\hline & 50 & 25 & 25 & 1.33 & 48.7 & 36.66 & - \\
\hline & 50 & 35 & 15 & 1.3 & 49.95 & 38.4 & - \\
\hline 1100 & 50 & 15 & 35 & 1.23 & 47 & 38 & - \\
\hline & 50 & 25 & 25 & 1.27 & 44.6 & 35 & - \\
\hline & 50 & 35 & 15 & 1.32 & 43.4 & 32.78 & 18.4 \\
\hline & & & & & & & \\
\hline 1200 & 50 & 15 & 35 & 1.24 & 49.8 & 40 & 1.22 \\
\hline & 50 & 25 & 25 & 1.25 & 47.7 & 38 & 6.14 \\
\hline & 50 & 35 & 15 & 1.37 & 47 & 34 & \\
\hline & & & & & & & 2.737 \\
\hline 1250 & 50 & 15 & 35 & 1.4 & 47 & 33.8 & 0.2 \\
\hline & 50 & 25 & 25 & 1.44 & 44.4 & 30.8 & 2.22 \\
\hline & 50 & 35 & 15 & 1.43 & 40 & 28 & 9.2 \\
\hline
\end{tabular}

\section{Table (3) :Mixture of clay and $\mathrm{Al}(\mathrm{OH}) 3$}

\begin{tabular}{|c|c|c|c|c|c|c|c|}
\hline $\begin{array}{c}\text { Firing } \\
\text { Temp } \\
\text { C. }\end{array}$ & $\begin{array}{c}\text { Content } \\
\text { of clay } \\
\%\end{array}$ & $\begin{array}{c}\text { Content } \\
\text { of burn- } \\
\text { ing clay } \\
\%\end{array}$ & $\begin{array}{c}\text { Content } \\
\text { of } \\
\mathrm{Al}(\mathrm{OH}) 3 \\
\%\end{array}$ & $\begin{array}{c}\text { Bulk } \\
\text { density }\end{array}$ & $\begin{array}{c}\text { Porosity } \\
\%\end{array}$ & $\begin{array}{c}\text { Water ab- } \\
\text { sorption } \\
\%\end{array}$ & $\begin{array}{c}\text { Compressive } \\
\text { strength } \\
\mathrm{kg} / \mathrm{cm} 2\end{array}$ \\
\hline 900 & 50 & 15 & 35 & - & - & - & - \\
\hline & 50 & 25 & 25 & - & - & - & - \\
\hline & 50 & 35 & 15 & 1.462 & 41.7 & 28.5 & - \\
\hline 1000 & 50 & 15 & 35 & - & - & - & - \\
\hline & 50 & 25 & 25 & - & - & - & - \\
\hline & 50 & 35 & 15 & 1.36 & 49 & 35.98 & - \\
\hline 1100 & 50 & 15 & 35 & - & - & - & - \\
\hline & 50 & 25 & 25 & 1.3 & 46.79 & 35.92 & 12.28 \\
\hline & 50 & 35 & 15 & - & - & - & - \\
\hline & & & & & & & 1.2 \\
\hline 1200 & 50 & 15 & 35 & 1.33 & 50.7 & 37.9 & 2.4 \\
\hline & 50 & 25 & 25 & 1.34 & 47.73 & 35.52 & 0.6 \\
\hline & 50 & 35 & 15 & 1.34 & 43 & 32 & \\
\hline & & & & & & & 2.4 \\
\hline 1300 & 50 & 15 & 35 & 1.2 & 44 & 37.4 & 0.7 \\
\hline & 50 & 25 & 25 & 2.2 & 13.8 & 6.2 & \\
\hline & 50 & 35 & 15 & 1.4 & 38.8 & 27.9 & 0.4 \\
\hline
\end{tabular}




\section{Table (4) :Mixture of clay and AIF3}

\begin{tabular}{|c|c|c|c|c|c|c|c|}
\hline $\begin{array}{c}\text { Firing } \\
\text { Temp } \\
\text { C. }\end{array}$ & $\begin{array}{c}\text { Content } \\
\text { of clay } \\
\%\end{array}$ & $\begin{array}{c}\text { Content } \\
\text { of burn- } \\
\text { ing clay } \\
\%\end{array}$ & $\begin{array}{c}\text { Content } \\
\text { of } \\
\mathrm{Al}(\mathrm{F}) 3 \\
\%\end{array}$ & $\begin{array}{c}\text { Bulk } \\
\text { density }\end{array}$ & $\begin{array}{c}\text { Porosity } \\
\%\end{array}$ & $\begin{array}{c}\text { Water ab- } \\
\text { sorption } \\
\%\end{array}$ & $\begin{array}{c}\text { Compressive } \\
\text { strength } \\
\mathrm{kg} / \mathrm{cm} 2\end{array}$ \\
\hline 900 & 50 & 15 & 35 & 1.05 & 60 & 56.7 & 12.28 \\
\hline & 50 & 25 & 25 & 1.22 & 53 & 43.4 & 18.43 \\
\hline & 50 & 35 & 15 & 1.4 & 46 & 33.3 & 18.43 \\
\hline 1000 & 50 & 15 & 35 & 0.97 & 62.6 & 64.6 & 36.86 \\
\hline & 50 & 25 & 25 & 1.14 & 53.5 & 46.97 & 61.44 \\
\hline & 50 & 35 & 15 & 1.35 & 46.9 & 34.46 & 63.9 \\
\hline 1100 & 50 & 15 & 35 & 0.89 & 60.37 & 67.36 & 33.8 \\
\hline & 50 & 25 & 25 & 1.1 & 53.3 & 48.7 & 43 \\
\hline & 50 & 35 & 15 & 1.27 & 46.5 & 36 & 49 \\
\hline 1200 & 50 & 15 & 35 & 0.933 & 63 & 67 & 32 \\
\hline 1200 & 50 & 15 & 35 & 1.15 & 55 & 47.68 & 64.5 \\
\hline & 50 & 25 & 25 & 1.4 & 50.32 & 36 & 43 \\
\hline & & & & & & & \\
\hline & & & & & & & \\
\hline 1250 & 50 & 15 & 35 & 1.13 & 55.2 & 48.8 & 22.1 \\
\hline & 50 & 25 & 25 & 1.37 & 46.3 & 33.64 & 43 \\
\hline & 50 & 35 & 15 & 1.4 & 36 & 25.73 & 43 \\
\hline
\end{tabular}

\section{Table (5): Mixture of clay and AIF3}

\begin{tabular}{|c|c|c|c|c|c|c|c|}
\hline $\begin{array}{c}\text { Firing } \\
\text { Temp } \\
\text { C. }\end{array}$ & $\begin{array}{c}\text { Content } \\
\text { of clay } \\
\%\end{array}$ & $\begin{array}{c}\text { Content } \\
\text { of burn- } \\
\text { ing clay } \\
\%\end{array}$ & $\begin{array}{c}\text { Content } \\
\text { of } \\
\mathrm{Al}(\mathrm{F}) 3 \\
\%\end{array}$ & $\begin{array}{c}\text { Bulk } \\
\text { density }\end{array}$ & $\begin{array}{c}\text { Porosity } \\
\%\end{array}$ & $\begin{array}{c}\text { Water ab- } \\
\text { sorption } \\
\%\end{array}$ & $\begin{array}{c}\text { Compressive } \\
\text { strength } \\
\mathrm{kg} / \mathrm{cm} 2\end{array}$ \\
\hline 900 & 60 & --- & 40 & 1.01 & 60 & 59 & 30.7 \\
\hline & 50 & --- & 50 & 0.9 & 66.5 & 74 & 18.4 \\
\hline & & & & & & & \\
\hline 1000 & 60 & --- & 40 & 0.84 & 64.3 & 76 & 55.3 \\
\hline & 50 & --- & 50 & 0.69 & 63.7 & 92.3 & 24.5 \\
\hline & & & & & & & \\
\hline 1100 & 60 & --- & 40 & 0.88 & 63.3 & 71.2 & 37.8 \\
\hline & 50 & --- & 50 & 0.83 & 66 & 79 & 14.7 \\
\hline & & & & & & & \\
\hline 1200 & 60 & --- & 40 & 0.83 & 62.8 & 75.3 & 30.8 \\
\hline & 50 & --- & 50 & 0.67 & 62.5 & 92.8 & 30.7 \\
\hline 1250 & 60 & --- & 40 & 0.96 & 58.3 & 60.5 & 32 \\
\hline & 50 & --- & 50 & 0.83 & 63.4 & 76.3 & 18.4 \\
\hline
\end{tabular}


Table 6 : Thermal properties of Al203 mixture

\begin{tabular}{|c|c|c|c|c|c|c|}
\hline $\begin{array}{c}\text { Firing } \\
\text { Temp. } \\
\text { C }\end{array}$ & $\begin{array}{c}\text { Content } \\
\text { of clay }\end{array}$ & $\begin{array}{c}\text { Content } \\
\text { of } \\
\text { burning } \\
\text { clay \% }\end{array}$ & $\begin{array}{c}\text { Content } \\
\text { of } \\
\text { Al2O3 }\end{array}$ & $\begin{array}{c}\text { Thermal } \\
\text { conductivity } \\
\text { kcal/mhc }\end{array}$ & $\begin{array}{c}\text { Heat sto- } \\
\text { rage ca- } \\
\text { pacity } \\
\text { kcal/c.m3 }\end{array}$ & $\begin{array}{c}\text { Heat dif- } \\
\text { fusivity } \\
\text { m2/h }\end{array}$ \\
\hline 900 & 50 & 15 & 35 & 0.326 & 260 & 0.00125 \\
\hline & 50 & 25 & 25 & 0.34 & 268 & 0.00126 \\
\hline & 50 & 35 & 15 & 0.35 & 274 & 0.00126 \\
\hline 1000 & 50 & 15 & 35 & 0.32 & 248 & 0.00129 \\
\hline & 50 & 25 & 25 & 0.345 & 256 & 0.001297 \\
\hline & 50 & 35 & 15 & 0.36 & 280 & 0.001285 \\
\hline 1100 & 50 & 15 & 35 & 0.31 & 246 & 0.00126 \\
\hline & 50 & 25 & 25 & 0.325 & 254 & 0.001279 \\
\hline & 50 & 35 & 15 & 0.333 & 264 & 0.001261 \\
\hline 1200 & 50 & 15 & 35 & 0.33 & 248 & 0.00129 \\
\hline & 50 & 25 & 25 & 0.322 & 250 & 0.001288 \\
\hline & 50 & 35 & 15 & 0.35 & 274 & 0.00127 \\
\hline 1250 & 50 & 15 & 35 & 0.36 & 280 & 0.001285 \\
\hline & 50 & 25 & 25 & 0.367 & 288 & 0.001274 \\
\hline & 50 & 35 & 15 & 0.366 & 286 & 0.001279 \\
\hline
\end{tabular}


Table 7 : Thermal properties of $\mathrm{Al}(\mathrm{OH}) 3$ mixture

\begin{tabular}{|c|c|c|c|c|c|c|}
\hline $\begin{array}{c}\text { Firing } \\
\text { Temp. } \\
\text { C }\end{array}$ & $\begin{array}{c}\text { Content } \\
\text { of clay } \\
\%\end{array}$ & $\begin{array}{c}\text { Content } \\
\text { of } \\
\text { burning } \\
\text { clay }\end{array}$ & $\begin{array}{c}\text { Content } \\
\text { of } \\
\mathrm{Al}(\mathrm{OH}) 3 \\
\%\end{array}$ & $\begin{array}{c}\text { Thermal } \\
\text { conductivity } \\
\mathrm{kcal} / \mathrm{m} . h . c\end{array}$ & $\begin{array}{c}\text { Heat sto- } \\
\text { rage ca- } \\
\text { pacity } \\
\text { kcal/c.m3 }\end{array}$ & $\begin{array}{c}\text { Heat dif- } \\
\text { fusivity } \\
\mathrm{m} 2 / \mathrm{h}\end{array}$ \\
\hline 900 & 50 & 15 & 35 & ---- & --- & --- \\
\hline & 50 & 25 & 25 & ---- & --- & --- \\
\hline & 50 & 35 & 15 & 0.38 & 292.4 & 0.00129 \\
\hline 1000 & 50 & 15 & 35 & --- & --- & --- \\
\hline & 50 & 25 & 25 & --- & --- & --- \\
\hline & 50 & 35 & 15 & 0.348 & 272 & 0.001279 \\
\hline 1100 & 50 & 15 & 35 & --- & --- & --- \\
\hline & 50 & 25 & 25 & 0.326 & 260 & 0.001253 \\
\hline & 50 & 35 & 15 & --- & --- & --- \\
\hline 1200 & 50 & 15 & 35 & 0.345 & 266 & 0.001297 \\
\hline & 50 & 25 & 25 & 0.3459 & 268 & 0.00129 \\
\hline & 50 & 35 & 15 & 0.3459 & 268 & 0.00129 \\
\hline 1300 & 50 & 15 & 35 & 0.3057 & 240 & 0.001273 \\
\hline & 50 & 25 & 25 & ---- & --- & --- \\
\hline & 50 & 35 & 15 & 0.36 & 280 & 0.001285 \\
\hline
\end{tabular}

Table 8: Thermal properties of AlF3 mixture

\begin{tabular}{|c|c|c|c|c|c|c|}
\hline $\begin{array}{c}\text { Firing } \\
\text { Temp. } \\
\text { C }\end{array}$ & $\begin{array}{c}\text { Content } \\
\text { of clay } \\
\%\end{array}$ & $\begin{array}{c}\text { Content } \\
\text { of } \\
\text { burning } \\
\text { clay }\end{array}$ & $\begin{array}{c}\text { Content } \\
\text { of AlF3 } \\
\%\end{array}$ & $\begin{array}{c}\text { Thermal } \\
\text { conductivity } \\
\text { kcal/m.h.c }\end{array}$ & $\begin{array}{c}\text { Heat sto- } \\
\text { rage ca- } \\
\text { pacity } \\
\text { kcal/c.m3 }\end{array}$ & $\begin{array}{c}\text { Heat dif- } \\
\text { fusivity } \\
\mathrm{m} 2 / \mathrm{h}\end{array}$ \\
\hline 900 & 50 & 15 & 35 & 0.235 & 194 & 0.001283 \\
\hline & 50 & 25 & 25 & 0.285 & 228 & 0.00125 \\
\hline & 50 & 35 & 15 & 0.3425 & 270 & 0.001268 \\
\hline 1000 & 50 & 15 & 35 & 0.255 & 210 & 0.001214 \\
\hline & 50 & 25 & 25 & 0.308 & 244 & 0.001262 \\
\hline & 50 & 35 & 15 & 0.36 & 280 & 0.001285 \\
\hline 1100 & 50 & 15 & 35 & 0.22 & 179.2 & 0.001227 \\
\hline & 50 & 25 & 25 & 0.275 & 220 & 0.00125 \\
\hline & 50 & 35 & 15 & 0.325 & 254 & 0.001279 \\
\hline 1200 & 50 & 15 & 35 & 0.225 & 186.6 & 0.001205 \\
\hline & 50 & 25 & 25 & 0.295 & 230 & 0.001282 \\
\hline & 50 & 35 & 15 & 0.36 & 280 & 0.001285 \\
\hline 1300 & 50 & 15 & 35 & 0.285 & 226 & 0.001261 \\
\hline & 50 & 25 & 25 & 0.35 & 274 & 0.001277 \\
\hline & 50 & 35 & 15 & 0.36 & 280 & 0.001285 \\
\hline
\end{tabular}


Table 9: Thermal properties of AlF3 mixture without burning clay

\begin{tabular}{|c|c|c|c|c|c|c|}
\hline $\begin{array}{c}\text { Firing } \\
\text { Temp. } \\
\text { C }\end{array}$ & $\begin{array}{c}\text { Content } \\
\text { of clay } \\
\%\end{array}$ & $\begin{array}{c}\text { Content } \\
\text { of } \\
\text { burning } \\
\text { clay }\end{array}$ & $\begin{array}{c}\text { Content } \\
\text { of AlF3 } \\
\%\end{array}$ & $\begin{array}{c}\text { Thermal } \\
\text { conductivity } \\
\mathrm{kcal} / \mathrm{m} . \mathrm{h} . \mathrm{c}\end{array}$ & $\begin{array}{c}\text { Heat sto- } \\
\text { rage ca- } \\
\text { pacity } \\
\mathrm{kcal} / \mathrm{c} . \mathrm{m} 3\end{array}$ & $\begin{array}{c}\text { Heat dif- } \\
\text { fusivity } \\
\mathrm{m} 2 / \mathrm{h}\end{array}$ \\
\hline 900 & 60 & & 40 & 0.25 & & \\
\hline & 50 & & 50 & 0.221 & & \\
\hline 1000 & 60 & & 40 & 0.195 & & \\
\hline & 50 & & 50 & 0.1575 & & \\
\hline & & & & & & \\
\hline 1100 & 60 & & 40 & 0.215 & & \\
\hline & 50 & & 50 & 0.2 & & \\
\hline & & & & & & \\
\hline 1200 & 60 & & 40 & 0.2 & & \\
\hline & 50 & & 50 & 0.15 & & \\
\hline & 50 & & & & & \\
\hline 1250 & 60 & & 40 & 0.1573 & & \\
\hline & 50 & & 50 & 0.2 & & \\
\hline
\end{tabular}

Table 10: Specifications of some building materials

\begin{tabular}{|c|c|c|c|c|c|}
\hline Material & $\begin{array}{c}\text { Bulk } \\
\text { density } \\
\mathrm{kg} / \mathrm{m} 3\end{array}$ & $\begin{array}{c}\text { Specific } \\
\text { heat } \\
\mathrm{kcal} / \mathrm{m} . \mathrm{h} . \mathrm{c}\end{array}$ & $\begin{array}{c}\text { Thermal } \\
\text { conductivity } \\
\mathrm{kcal} / \mathrm{m} . \mathrm{h} . \mathrm{c}\end{array}$ & $\begin{array}{c}\text { Heat storage } \\
\text { capacity } \\
\mathrm{kcal} / \mathrm{c} . \mathrm{m} 3\end{array}$ & $\begin{array}{c}\text { Heat dif- } \\
\text { fusivity } \\
\mathrm{m} 2 / \mathrm{h}\end{array}$ \\
\hline $\begin{array}{c}\text { Light weight } \\
\text { refractory }\end{array}$ & 670 & 0.2 & 0.15 & 134 & 0.0011194 \\
\hline $\begin{array}{c}\text { Light weight } \\
\text { brick }\end{array}$ & 900 & 0.2 & 0.32 & 180 & 0.00122 \\
\hline Heavy brick & 1500 & 0.2 & 0.5 & 300 & 0.00167 \\
\hline Thermostone & 800 & 0.22 & 0.23 & 176 & 0.00131 \\
\hline $\begin{array}{c}\text { Heavy con- } \\
\text { crete }\end{array}$ & 2300 & 0.2 & 1.75 & 460 & 0.0038 \\
\hline Styropore & 20 & 0.33 & 0.03 & 6.6 & 0.00455 \\
\hline
\end{tabular}




\begin{tabular}{|c|c|}
\hline 0 & -5 \\
\hline 100 & 0.3 \\
\hline 500 & 2 \\
\hline 580 & 0 \\
\hline 900 & -0.15 \\
\hline 1050 & 2.8 \\
\hline 1100 & -3.2 \\
\hline
\end{tabular}

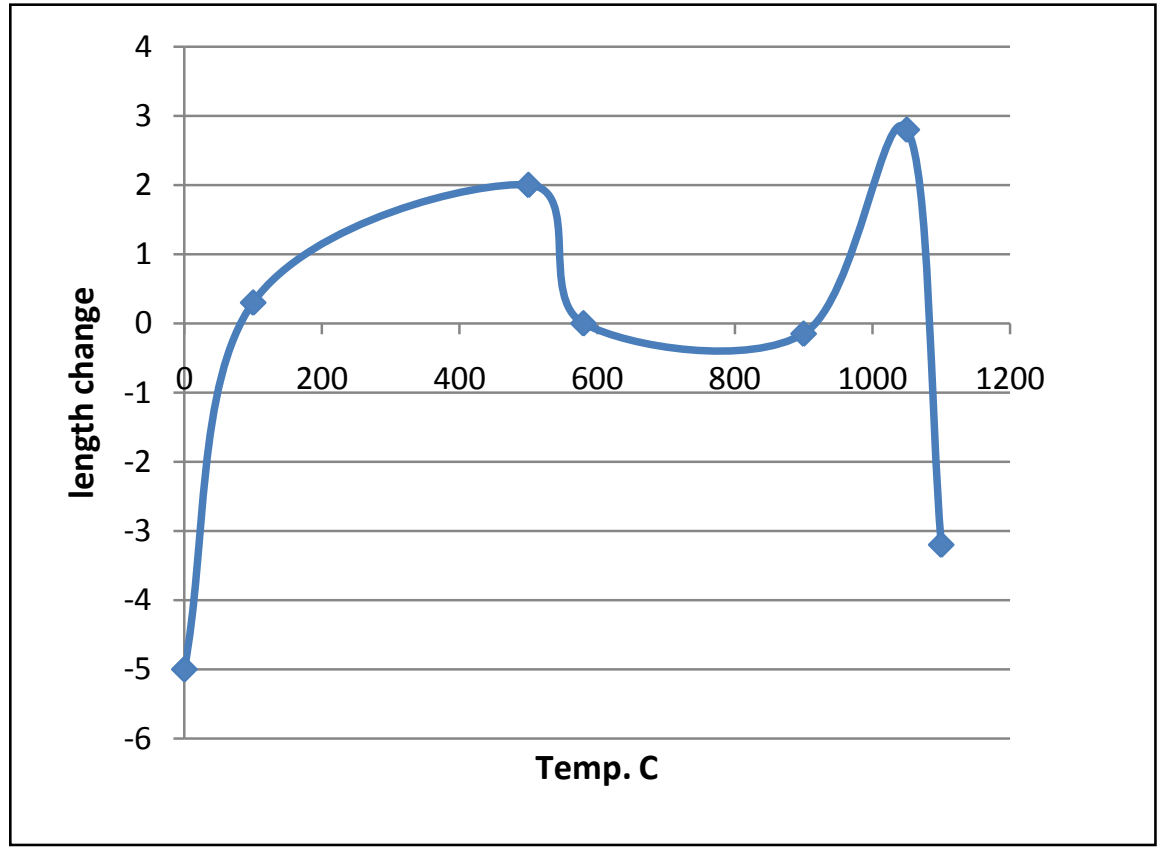

Fig. 1: relationship between length change $\%$ and temperature 


\begin{tabular}{|c|c|}
\hline 800 & 0.8 \\
\hline 900 & 1.05 \\
\hline 1000 & 1 \\
\hline 1100 & 0.8 \\
\hline 1200 & 0.93 \\
\hline 1300 & 1.12 \\
\hline
\end{tabular}

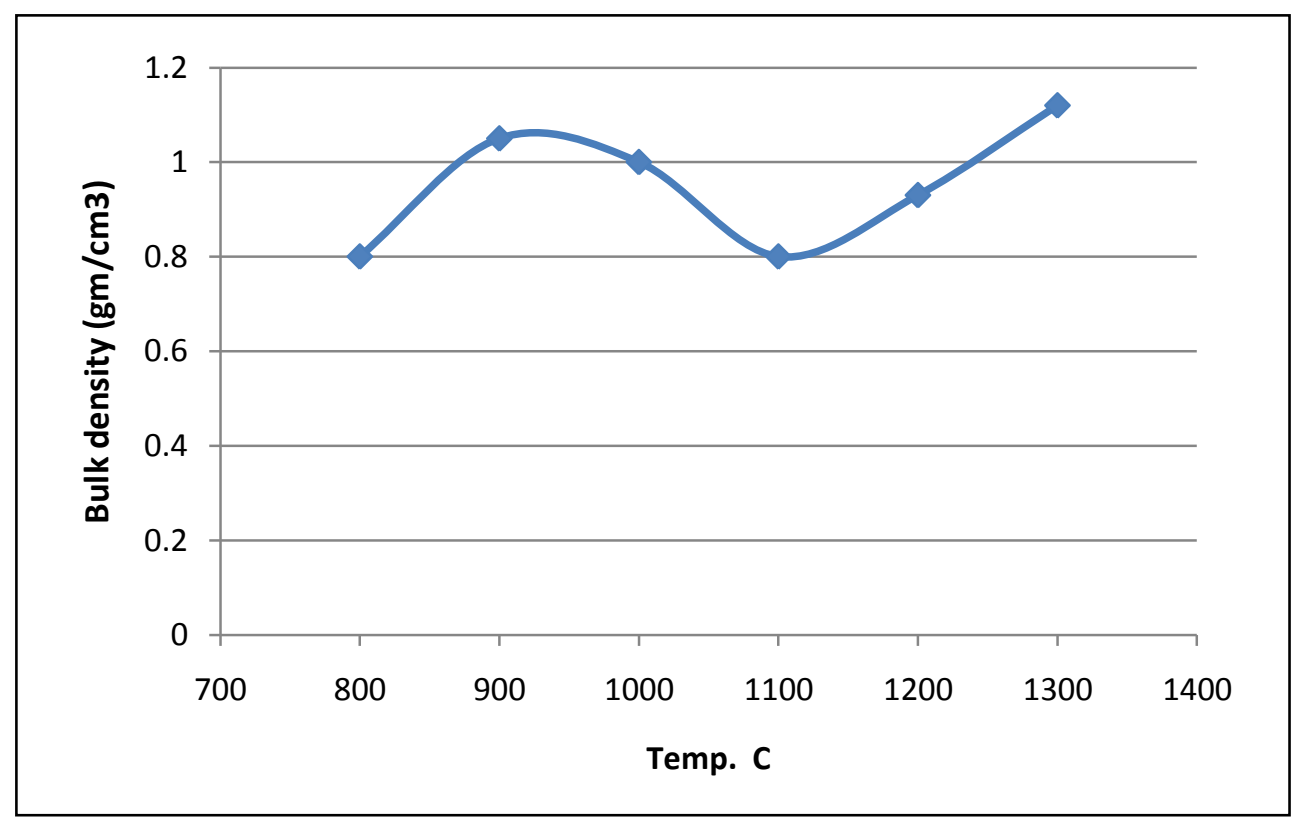

Fig .2: relationship between bulk density temperature 


\begin{tabular}{|c|c|}
\hline 800 & 50 \\
\hline 900 & 60 \\
\hline 1000 & 62.5 \\
\hline 1100 & 60.5 \\
\hline 1250 & 55 \\
\hline
\end{tabular}

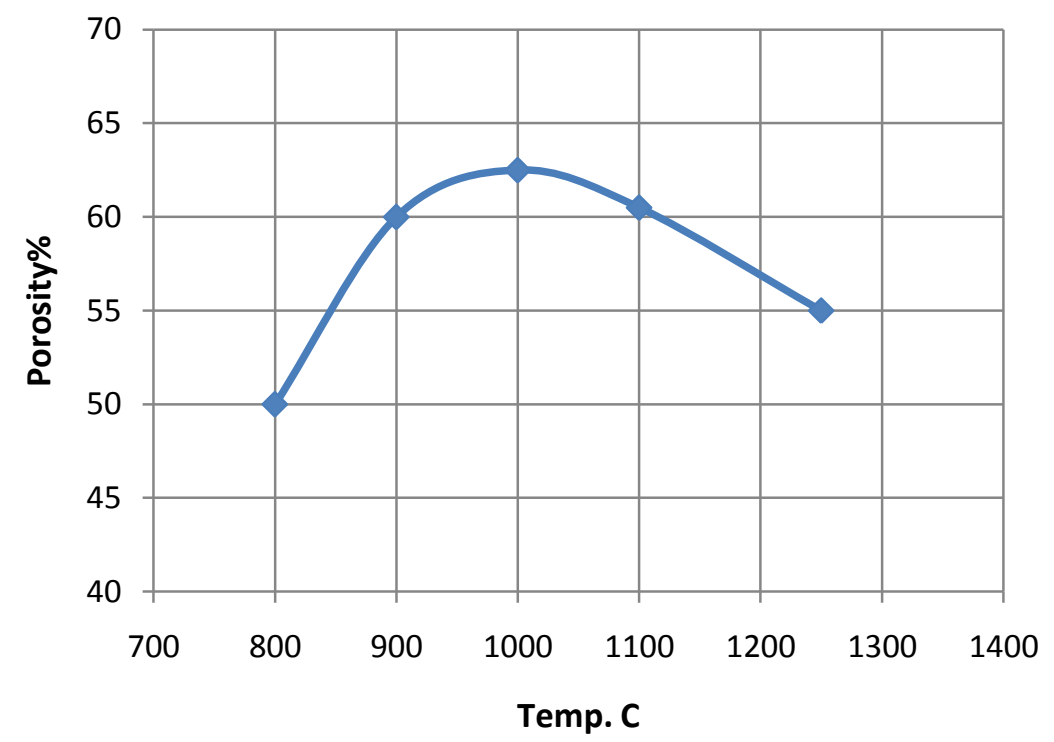

Fig.3: relationship between Porosity \% temperature 


\begin{tabular}{|c|c|}
\hline 800 & 10 \\
\hline 900 & 12.2 \\
\hline 1000 & 36.6 \\
\hline 1100 & 34 \\
\hline 1200 & 32.6 \\
\hline 1300 & 23 \\
\hline
\end{tabular}

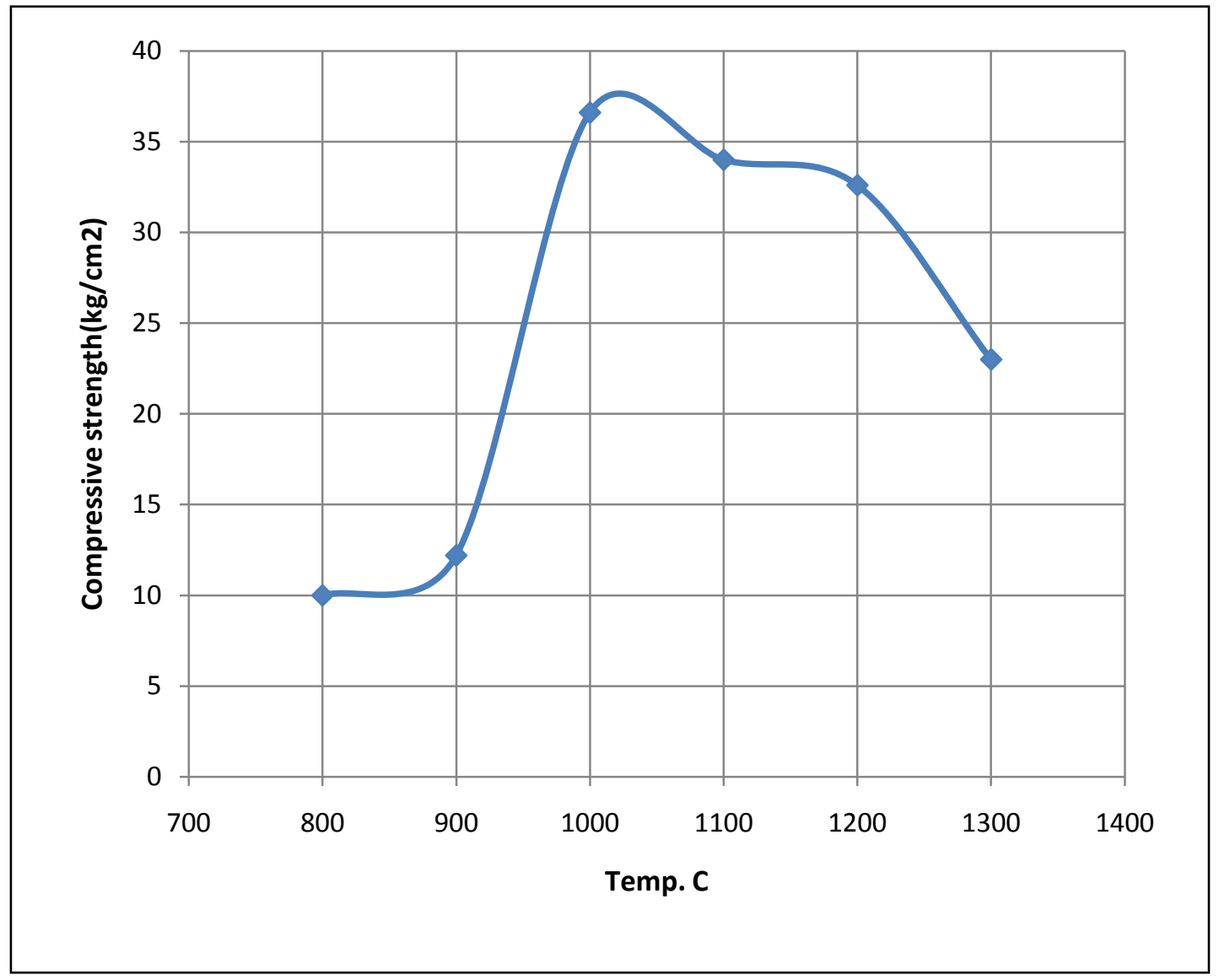

Fig.4: relationship between Compressive strength $\mathrm{kg} / \mathrm{cm} 2$ temperature 


\begin{tabular}{|c|c|}
\hline 500 & 0 \\
\hline 700 & 0.15 \\
\hline 840 & 0.2 \\
\hline 1200 & 0.3 \\
\hline 1500 & 0.4 \\
\hline
\end{tabular}

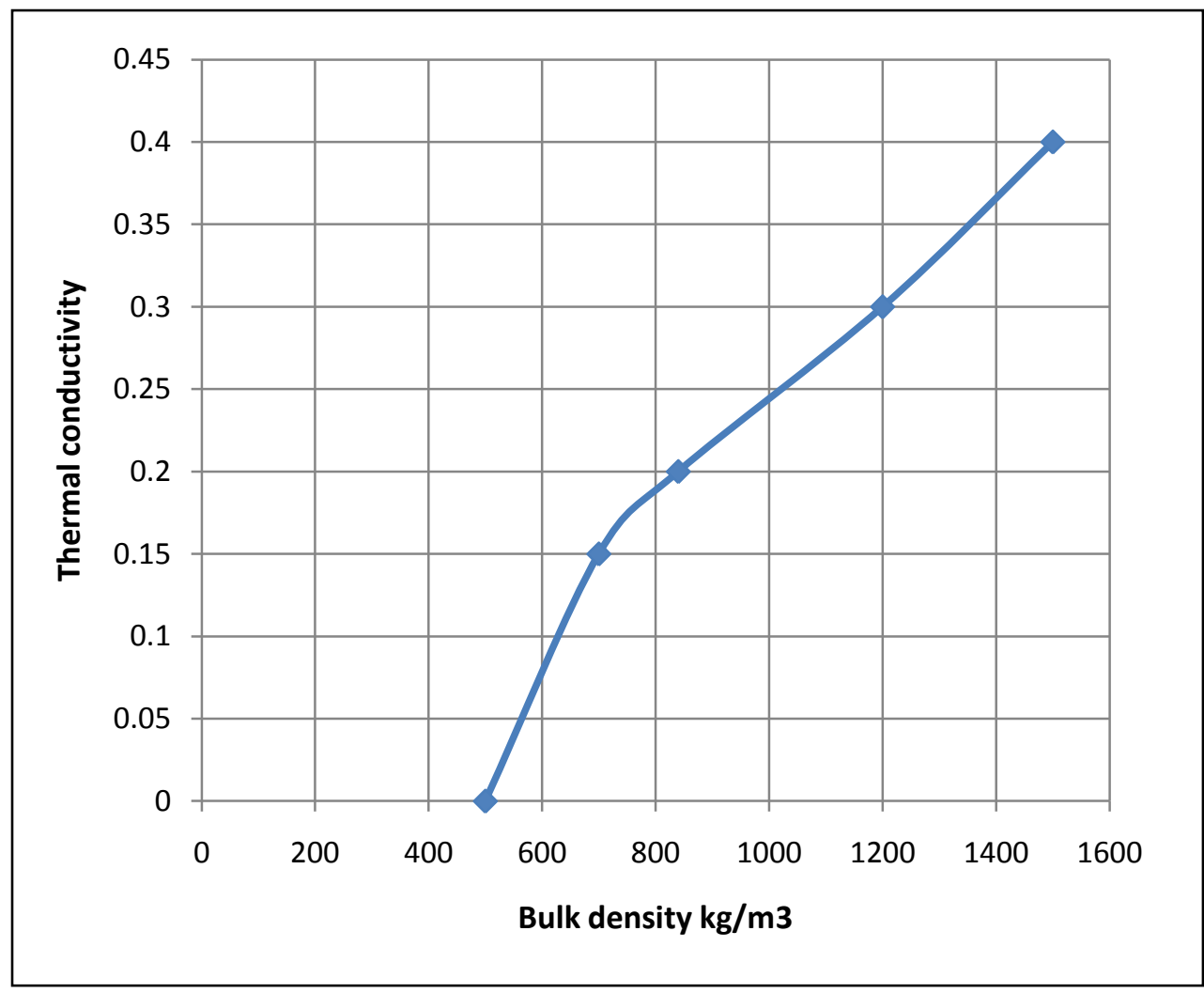

Fig.5: Correlation graph for light weight refractory 


\begin{tabular}{|c|c|c|}
\hline 0 & 20 & 16 \\
\hline & & \\
\hline 1.8 & 24 & \\
\hline 2 & & 17 \\
\hline 3 & 27 & 18 \\
\hline 4.5 & & 21 \\
\hline 6 & 36 & 25 \\
\hline 7 & 38 & \\
\hline 7.6 & & 30 \\
\hline
\end{tabular}

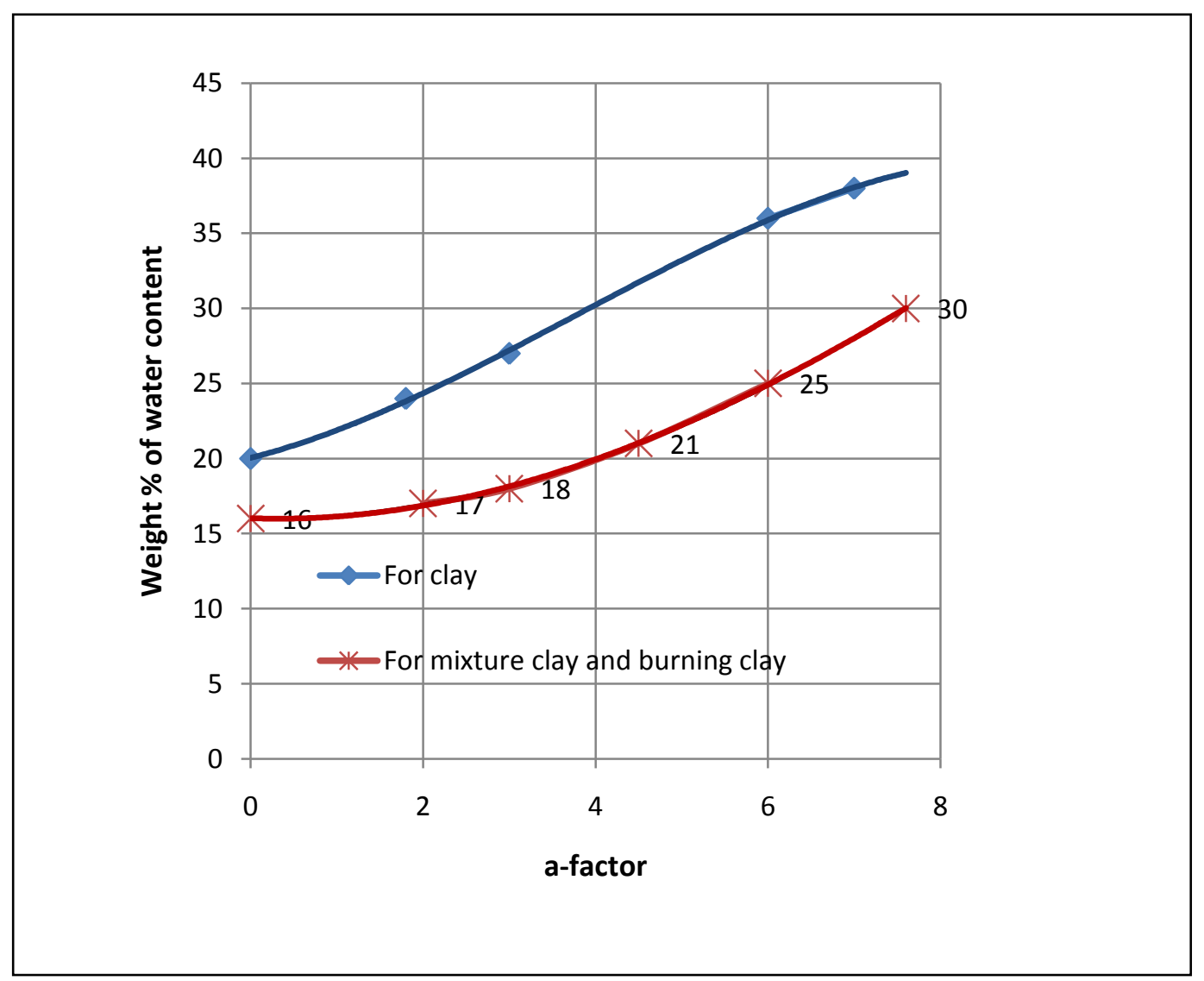

Fig.6: relationship between weight $\%$ of water content and a- factor 


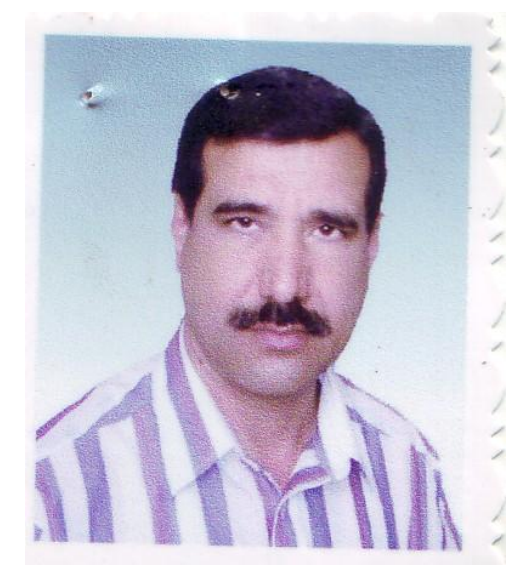

$$
\text { الاسم : عبدالكريم دهش عفات الزبيدي }
$$

$$
\text { موقع العمل :استاذ في كلية الهندسة /جامعة النهرين - بغداد }
$$

التحصيل الدراسي : ماجستير هكسة كيماوي (وحدات صناعية) في الجامعة التكنولوجيا

بغاد/1983 